\title{
900 nm Emission of a Nd:ASL Laser Pumped by an Extended-Cavity Tapered Laser Diode
}

\author{
D. Paboeuf, G. Lucas-Leclin, P. Georges \\ Laboratoire Charles Fabry de l'Institut d'Optique, UMR 8501 \\ Campus Polytechnique, RD128, F-91127 Palaiseau Cedex, France \\ david.paboeuf@institutoptique.fr \\ B. Sumpf, G. Erbert \\ Ferdinand-Braun-Institut für Höchstfrequenztechnik, \\ Albert-Einstein-Strasse 11, 12489 Berlin, Germany \\ C. Varona, P. Loiseau, G. Aka \\ Laboratoire de Chimie de la Matière Condensée de Paris, CNRS-UMR 7574 \\ ENSCP, 11 rue P. et M. Curie, F-75231 Paris Cedex 05, France \\ B. Ferrand \\ Laboratoire de Cristallogenèse Appliquée, CEA - LETI \\ 17 Rue des martyrs, F-38054 Grenoble Cedex, France
}

\begin{abstract}
We describe here the use of a 798-nm-stabilized high-brightness tapered laser diode to pump
\end{abstract} a Nd:ASL crystal for $900 \mathrm{~nm}$ laser operation. An output power of $150 \mathrm{~mW}$ is obtained.

\section{Introduction}

Neodymium-doped laser materials, operating on the ${ }^{4} \mathrm{~F}_{3 / 2} \rightarrow{ }^{4} \mathrm{I}_{9 / 2}$ laser transition around $900 \mathrm{~nm}$, have a great interest for blue laser generation by frequency doubling in a quadratic non-linear optical crystal. Efficient quasithree-level emission has been obtained in various laser materials, such as Nd:YAG $(946 \mathrm{~nm}), \mathrm{Nd}: \mathrm{YVO}_{4}$ $(914 \mathrm{~nm}), \mathrm{Nd}: \mathrm{GdVO}_{4}(912 \mathrm{~nm})$ [1-3]. However, the blue wavelength obtained by frequency doubling of these lasers is not short enough for some applications.

The Nd-doped strontium-lanthanum aluminate crystal: $\mathrm{Nd}_{0.05} \mathrm{Sr}_{0.7} \mathrm{La}_{0.25} \mathrm{Mg}_{0.3} \mathrm{Al}_{11.7} \mathrm{O}_{19}$, called Nd:ASL, could be a solution since its ${ }^{4} \mathrm{~F}_{3 / 2} \rightarrow{ }^{4} \mathrm{I}_{9 / 2}$ transition correspond to a laser line at $900 \mathrm{~nm}$. Unfortunately, the absorption transitions of Nd:ASL are narrowband and located at $792 \mathrm{~nm}$ and $798 \mathrm{~nm}$ where no commercial high power laser diodes are available. Then, Nd:ASL laser operation at $900 \mathrm{~nm}$ has only been obtained under Ti:Sa pumping at $792 \mathrm{~nm}$ [4]. Moreover, the quasi-three-level operation of this laser requires a high-brightness pump source to exceed the transparency intensity all along the crystal. Thus we propose a novel external-cavity design based on a high-brightness tapered amplifier diode. This laser diode was wavelength stabilized at $798 \mathrm{~nm}$, closer to the maximum-gain wavelength of the tapered amplifier, by use of a volume Bragg grating (VBG) in a simple and compact set-up.

We report here the design and the characteristics of our extended-cavity pump source and the use of this high-brightness and narrow laser source to pump a crystal of Nd:ASL for laser operation at $900 \mathrm{~nm}$.

\section{Description and Characterization of the pumping source}

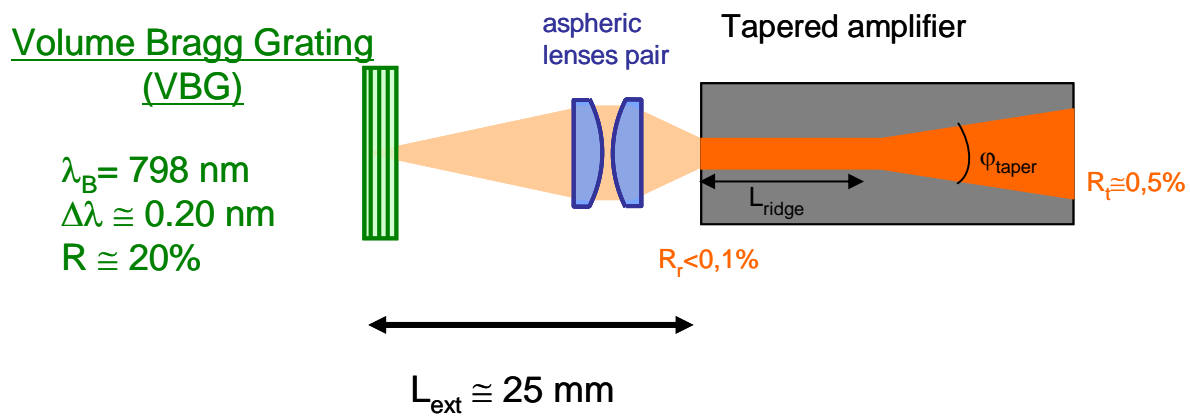

Figure 1 : Design of the extended-cavity tapered laser with a Bragg grating.

Tapered laser diode is a promising design for high-brightness emission in the 1 to $5 \mathrm{~W}$ power range. It consists in a single-mode ridge acting as a spatial filter and a tapered amplifier section [5]. The one we used had an emission centered around $800 \mathrm{~nm}$ and was designed and grown at Ferdinand Braun Institut für Höchsfrequenztechnick. The active region was composed of a tensile-strained GaAsP single quantum well embedded in a 3- $\mu \mathrm{m}$ thick $\mathrm{Al}_{0.45} \mathrm{Ga}_{0.55} \mathrm{As}$ waveguide and highly doped $\mathrm{Al}_{0.7} \mathrm{Ga}_{0.3}$ As cladding layers [6]. The index-guided straight ridge section is 2 -mm-long and the gain-guided tapered section is characterized by a 
tapered angle of $4^{\circ}$ and a length of $2 \mathrm{~mm}$. The front facet of the tapered laser has a reflectivity of $0.5 \%$, whereas the rear facet reflectivity was about $0.1 \%$. A passivation coating has been added prior to the antireflection one for improved facet stability.

We mounted the tapered amplifier in an extended cavity with a volume Bragg grating acting as a back mirror. The grating is characterized by a Bragg wavelength $\lambda_{B}=798 \mathrm{~nm}$, a reflectivity of $20 \%$ and a spectral selectivity of $0.2 \mathrm{~nm}$. The emission from the ridge side is focused on the grating by a high-numerical-aperture aspheric lenses pair (Fig. 1). This configuration result in a lower sensitivity to misalignements compared to the collimated-on-the-grating standard one and also in a better control of the emitted wavelength [7].

Without any feedback from the grating, no laser emission has been observed but only amplified spontaneous emission centered around $800 \mathrm{~nm}$ and covering a large band of spectrum. In the extended cavity, the laser emission is locked on the Bragg wavelength whatever the operating current or the temperature (Fig. 3) with a very low wavelength shift $(<200 \mathrm{pm})$. The side-mode suppression ratio is higher than $40 \mathrm{~dB}$. The laser FWHM-linewidth remains lower than $80 \mathrm{pm}$ (OSA-limited). The threshold of the laser diode was as low as $1 \mathrm{~A}$ and the slope efficiency reached $0.9 \mathrm{~W} . \mathrm{A}^{-1}$. We obtained a maximum output power of $1.4 \mathrm{~W}$ for an operating current of 2.5 A (Fig. 2), with no evidence of roll-over. These results are very close to those obtained with a tapered laser based on the same layer design but with a high reflective coating on the back facet [6], which proves that our extended cavity design doesn't limit the intrinsic performance of the tapered amplifier.

The output laser beam was collimated in the fast axis with an aspheric lens $\left(\mathrm{f}^{\prime}=8 \mathrm{~mm}\right)$. The strong astigmatism of the tapered laser was easily corrected with a cylindrical lens. The focal length of the cylindrical lens $\left(\mathrm{f}^{\prime}=300 \mathrm{~mm}\right)$ was chosen to circularize the output beam. We measured a beam quality parameter $\mathrm{M}^{2}$ as good as 1.2 in both directions for an operating current of $2 \mathrm{~A}$, which degrades to $\sim 3$ at higher operating current. The maximum brightness value is evaluated to $110 \mathrm{MW} \cdot \mathrm{cm}^{-2} \mathrm{sr}^{-1}$.

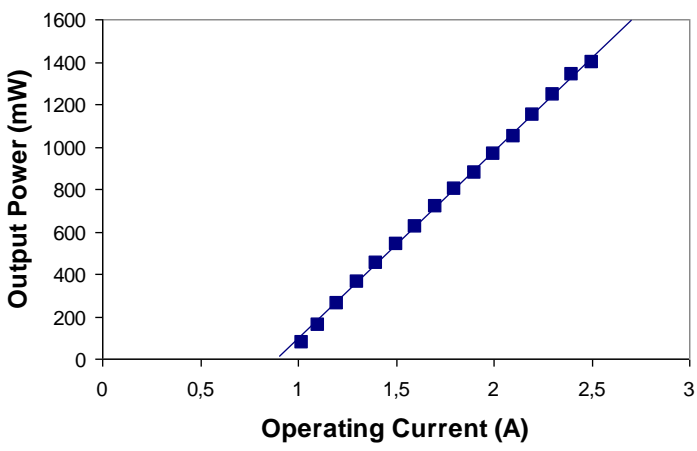

Figure 2 : Output power vs operating current; operating temperature $=25^{\circ} \mathrm{C}$

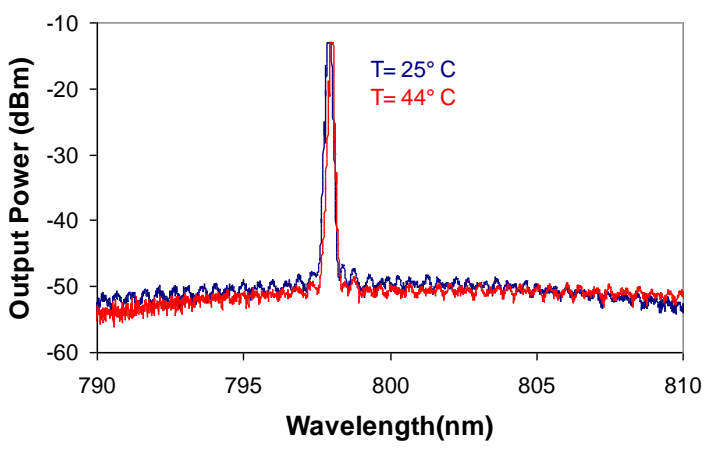

Figure 3 : Laser spectrum of the tapered laser in extended cavity, at different temperatures; operating current $=2 \mathrm{~A}$

\section{Laser operation of Nd:ASL at $900 \mathrm{~nm}$}

The CW $900 \mathrm{~nm}$ laser emission was investigated by using an end-pumped plane-concave resonator with a $\mathrm{Nd}$ :ASL crystal grown by the Czochralski pulling technique. The crystal was mounted on a copper block and maintained at a temperature of approximately $14^{\circ} \mathrm{C}$ by a water-cooling device. The input mirror was deposited on the first face of the crystal (high transmission at the pump wavelength and $1050 \mathrm{~nm}$, high reflection at 900 $\mathrm{nm})$. The 100-mm-curvature concave output mirror had a high reflectivity at $900 \mathrm{~nm}$ and a high transmission at $1050 \mathrm{~nm}$ to prevent laser emission from the intense ${ }^{4} \mathrm{~F}_{3 / 2} \rightarrow{ }^{4} \mathrm{I}_{11 / 2} 4$ level transition. For maximum absorption, the polarization of the pump beam was set perpendicular to the c-axis of the crystal by a half-wave plate. We also used an isolator in order to protect the tapered laser from parasitic back-reflections. The pump beam was focused inside the crystal by a lens.

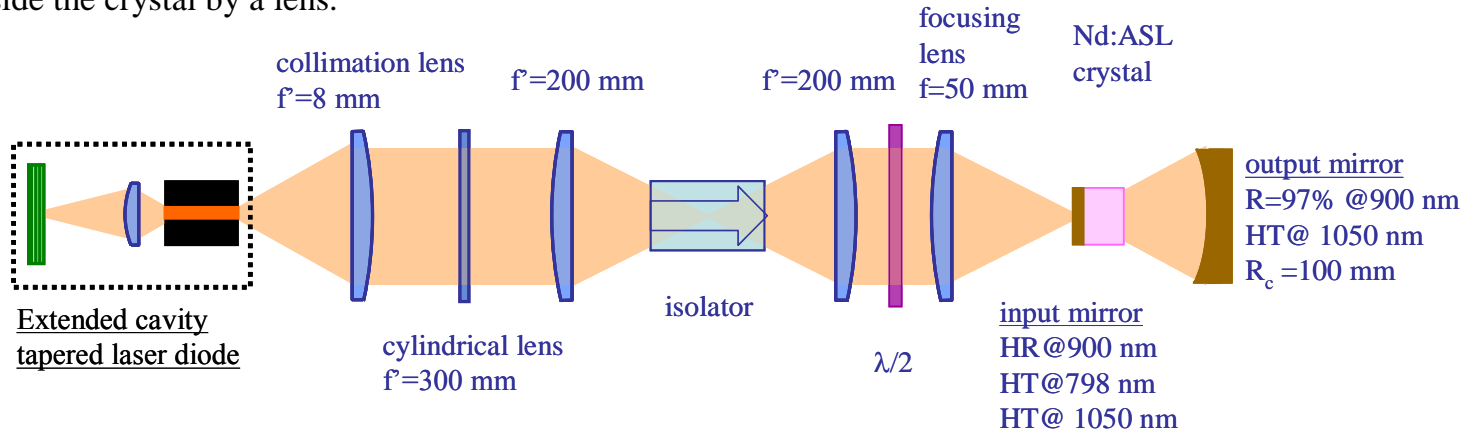

Figure 4 : Experimental setup 
Several output mirrors with different transmissions at $900 \mathrm{~nm}$ have been tested. The optimum pump waist diameter has also been investigated by changing the focal length of the focusing lens. The highest power has been obtained with a $\mathrm{T}=3 \%$ output mirror and a pump waist diameter of $20 \mu \mathrm{m}$. A maximum output power of $150 \mathrm{~mW}$ for a $1.1 \mathrm{~W}$ incident pump power (560 mW absorbed) has been obtained with a 3-mm-long crystal (Fig.5). The laser threshold is observed at the incident power of $200 \mathrm{~mW}$, corresponding to an absorbed pump power of $120 \mathrm{~mW}$. This leads to an efficiency of $34 \%$ with the absorbed power. The laser emission is centered at $900.3 \mathrm{~nm}$ with a linewidth of $1 \mathrm{~nm}$. With a 5-mm-long crystal, lower output powers have been obtained. The crystal being longer, the pump intensity at the end of the crystal is lower than transparency intensity, resulting in an increased reabsorption at the laser wavelength. The degradation of the pump beam quality at high powers enhanced this effect.

Under Ti:Sa pumping, an absorbed pump power of $560 \mathrm{~mW}$ permitted to reach a power as high as $200 \mathrm{~mW}$ with a 5-mm-long crystal [4]. Our performance is then lower. The main reason is the degradation of the pump beam quality for high operating currents. This fact is confirmed by the beam quality factor $\mathrm{M}^{2}$ of the laser beam being 1.5 at maximum output power.

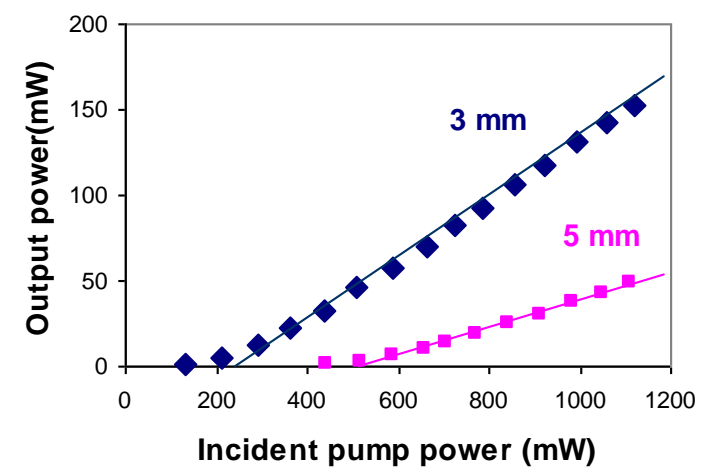

Figure 5 : Laser output power vs incident pump power, $R_{\text {ouput coupler }}=97 \%$, pump beam diameter $=20 \mu \mathrm{m}$

\section{Conclusion}

We have demonstrated what is, to our knowledge, the first diode-pumped Nd:ASL continuous-wave laser operating at $900 \mathrm{~nm}$. This is a great step on the way to a compact design for Nd:ASL-based blue lasers. A highbrightness tapered laser diode has been stabilized at $798 \mathrm{~nm}$ using a volume Bragg grating in a very compact extended cavity. Furthermore, This laser source has a high brightness and a narrow line, which makes it fully suitable for the pumping of Nd:ASL. A power of $1.5 \mathrm{~W}$ has been reached with this diode. We have used the tapered diode to pump a Nd:ASL crystal to obtain a maximum power of $150 \mathrm{~mW}$ at $900 \mathrm{~nm}$. Experiments of intracavity doubling will now be performed with a non-linear optical crystal in order to obtain a blue laser emission at $450 \mathrm{~nm}$.

Acknowledgment: this work has been partially supported by the European Community under the www.BRIGHT.eu integrated project. (IP511722)

\section{References}

[1] T. Y. Fan and al., "Continuous-wave operation of a room-temperature, diode-laser-pumped, 946-nm Nd:YAG laser”, Opt. Lett. 12, 809 (1987)

[2] P. Zeller and al., "Efficient, multiwatt, continuous-wave laser operation on the ${ }^{4} \mathrm{~F}_{3 / 2}-{ }^{4} \mathrm{I}_{9 / 2}$ transitions of $\mathrm{Nd}: \mathrm{YVO}_{4}$ and Nd:YAG", Opt. Lett. 25, 34-36 (2000)

[3] C. Czeranowsky and al., "Continuous wave diode pumped intracavity doubled $\mathrm{Nd}: \mathrm{GdVO}_{4}$ laser with $840 \mathrm{~mW}$ output power at $456 \mathrm{~nm}$ ", Opt. Commun. 205, 361-365 (2002)

[4] C. Varona and al., "CW blue laser emission by second harmonic generation of $900 \mathrm{~nm}$ oscillation of Nd-doped strontium and lanthanum aluminate (ASL)", Solid-State Lasers and Amplifiers, Proc. of SPIE, vol. 6190, 61900D

[5] H. Wenzel and al., "High Brightness diodes lasers", C. R. Physique 4 (2003)

[6] F. Dittmar and al., "High-Power 808-nm Tapered Diode Lasers With Nearly Diffraction-Limited Beam Quality of $\mathrm{M}^{2}=1.9$ at $\mathrm{P}=4.4 \mathrm{~W}$ ", IEEE Photonics Technology Letters 18, n4, 601-603

[7] G. Lucas-Leclin and al., "Wavelength Stabilization of Extended-Cavity Tapered Lasers with Volume Bragg Gratings", CLEO 2006 